\title{
RESTRICTIONS OF ANALYTIC FUNCTIONS. III
}

\author{
MARVIN ROSENBLUM AND JAMES ROVNYAK ${ }^{1}$
}

ABSTRACT. Characterizations are given of all real and all pure imaginary functions on a real Borel set which occur as restrictions of boundary functions of functions holomorphic and having positive imaginary part in the upper half-plane.

1. Introduction. Let $f$ be a function which is holomorphic and has positive imaginary part in the upper half-plane $\Pi_{+}$. Then $f$ is determined by the restriction of its boundary function to any real Borel set $\Delta$ of positive measure. Previous work [3], [4] has been concerned with characterizations of restrictions of this kind and a method by which the holomorphic function can be recaptured from a given restriction. To a large degree our work has been motivated by attempts to solve two problems concerning Hilbert transforms which arose out of [2]. Recall that if $f \in L^{2}(\Delta)$, then the Hilbert transform $\tau_{\Delta} f$ of $f$ is defined a.e. on $(-\infty, \infty)$ by

$$
\left(\tau_{\Delta} f\right)(x)=P V \frac{1}{\pi} \int_{\Delta} \frac{f(t)}{t-x} d t
$$

Problem I (Generalized Loewner Problem). Characterize all real valued measurable functions $u$ on $\Delta$ such that

$$
\frac{1}{\pi} \iint_{\Delta \times \Delta}^{\prime} \frac{u(x)-u(t)}{x-t} f(t) f(x)^{*} d t d x \geq 0
$$

for all $f \in L^{2}(\Delta)$ such that $u f \in L^{2}(\Delta)$.

The integral in (1) is taken as

$$
\begin{aligned}
\lim _{\epsilon>0} \frac{1}{\pi} \iint_{\Delta \times \Delta} \chi_{\{|x-t|>\epsilon\}}(x, t) & \frac{u(x)-u(t)}{x-t} f(t) f(x)^{*} d t d x \\
& =\left\langle\tau_{\Delta}(u f), f\right\rangle_{L^{2}(\Delta)}-\left\langle\tau_{\Delta} f, u f\right\rangle_{L^{2}(\Delta)} .
\end{aligned}
$$

Problem II. Characterize all nonnegative measurable functions $v$ on $\Delta$ such that

$$
\int_{\Delta}\left|\left(\tau_{\Delta} f\right)(t)\right|^{2} v(t) d t \leq \int_{\Delta}|f(t)|^{2} v(t) d t
$$

for all $f \in L^{2}(\Delta)$ such that $v f \in L^{2}(\Delta)$.

Received by the editors August 12, 1974. $44 \mathrm{~A} 25$.

AMS (MOS) subject classifications (1970). Primary 30A76; Secondary 30 A88,

Key words and phrases. Hilbert transform, analytic function with positive imaginary part, exponential representation.

${ }^{1}$ Research supported by NSF Grant GP 31483X. The first author al so acknowledges support from the Center for Advanced Studies of the University of Virginia. 
Quite remarkably, these problems have simple solutions involving functions in the class $R$ of functions holomorphic and having nonnegative imaginary part in $\Pi_{+}$. In [3] we showed that a real valued function $u$ on $\Delta$ is a solution to the generalized Loewner problem if and only if there exists $w$ in $R$ such that

$$
u(x)=w(x+i 0) \text { a.e. on } \Lambda .
$$

In this paper we show that a nonnegative function $v$ on $\Delta$ is a solution to Problem II if and only if there exists $w$ in $R$ such that

$$
i v(x)=w(x+i 0) \text { a.e. on } \Delta .
$$

2. Exponential representations. Let $\Delta$ be a real Borel set such that neither $\Delta$ nor its complement $\Delta^{c}$ is a Lebesgue null set. Let $U(\Delta)$ be the class of real valued functions $u$ on $\Delta$ of form (3) for some $w \in R$, and let $V(\Delta)$ be the class of nonnegative functions $v$ on $\Delta$ of form (4) for some $w \in R$.

In this section we collect three elementary results concerning exponential representations for functions in $U(\Delta)$ and $V(\Delta)$. From [1] we know that $w \in R$ if and only if $w$ has a representation of the form

$$
w(z)=C \exp \left(\int_{-\infty}^{+\infty} \frac{1+t z}{t-z} \frac{f(t)}{1+t^{2}} d t\right), \quad z \in \Pi_{+},
$$

where $C$ is a nonnegative constant and $f$ is a measurable function such that $0 \leq f \leq 1$ a.e. on $(-\infty, \infty)$. Then

$$
u(x+i 0)=C \exp \left(P V \int_{-\infty}^{+\infty} \frac{1+t x}{t-x} \frac{f(t)}{1+t^{2}} d t+\pi i f(x)\right)
$$

for almost all $x$ in $(-\infty, \infty)$.

From (5) and (6) we easily derive the following two theorems.

Theorem 1. Suppose $\dot{w} \in R$.

(i) $\operatorname{Im} w(x+i 0)=0$ a.e. on $\Delta$ if and only if (5) holds, where $C \geq 0$, $0 \leq f(t) \leq 1$ a.e. on $\Delta^{c}$, and $f(t)=\chi_{E}$ a.e. on $\Delta$ for some Borel subset $E$ of $\Delta$.

(ii) $\operatorname{Re} w(x+i 0)=0$ a.e. on $\Delta$ if and only if (5) holds, where $C \geq 0$, $0 \leq f(t) \leq 1$ a.e. on $\Delta^{c}$, and $f(t)=1 / 2$ a.e. on $\Delta$.

Theorem 2. (i) A real valued function $u$ on $\Delta$ is in $U(\Delta)$ if and only if $u$ is given a.e. on $\Delta$ by

$$
u(x)=C \exp \left(P V \int_{E} \frac{1+t x}{t-x} \frac{d t}{1+t^{2}}+i \pi \chi_{E}(x)\right) \exp \left(P V \int_{\Delta^{c}} \frac{1+t x}{t-x} \frac{f(t)}{1+t^{2}} d t\right)
$$

where $C \geq 0,0 \leq f(t) \leq 1$ a.e. on $\Delta^{c}$, and $E$ is a Borel subset of $\Delta$.

(ii) A nonnegative function $v$ on $\Delta$ belongs to $V(\Delta)$ if and only if $v$ is 
given a.e. on $\Delta$ by

$$
v(x)=C \exp \left(P V \frac{1}{2} \int_{\Delta} \frac{1+t x}{t-x} \frac{d t}{1+t^{2}}\right) \exp \left(P V \int_{\Delta^{c}} \frac{1+t x}{t-x} \frac{f(t)}{1+t^{2}} d t\right)
$$

where $C \geq 0$ and $0 \leq f(t) \leq 1$ a.e. on $\Delta^{c}$.

A bounded real valued function on $(-\infty, \infty)$ which occurs as the boundary function of an $H^{\infty}$ function is necessarily a constant a.e. In contrast, there exist many bounded real valued functions on $\Delta$ which are restrictions of boundary functions of $H^{\infty}$ functions.

Theorem 3. A bounded real valued function $f$ on $\Delta$ is the restriction of the boundary function of an $H^{\infty}$ function if and only if $f$ is given a.e. on $\Delta$ by

$$
f(x)=\lambda[v(x)-1] /[v(x)+1]
$$

where $\lambda$ is a real constant and $v \in V(\Delta)$.

Proof. This follows from the fact that $F \in H^{\infty}$ if and only if $F(z)=$ $\lambda[W(z)-i] /[W(z)+i]$ where $\lambda$ is a real constant and $W \in R$.

3. Main result. The following properties of $\tau_{\Delta}$ will be useful for our consideration of Problem II.

(i) Suppose $f \in L^{p}(\Delta)$ where $p>1$. Then $\tau_{\Delta} f \in L^{p}(\Delta)$ and $\tau_{\Delta}$ is a bounded operator on $L^{p}(\Delta)$ to $L^{p}(\Delta)$.

(ii) Suppose $f, g \in L^{2}(\Delta)$. Then

$$
\int_{\Delta}\left[\left(\tau_{\Delta} f\right)(t) g(t)^{*}+f(t)\left(\tau_{\Delta} g\right)(t)^{*}\right] d t=0 .
$$

(iii) Suppose $f \in L^{p}(\Delta), g \in L^{q}(\Delta)$ where $p^{-1}+q^{-1}<1$. Then

$$
\tau_{\Delta}\left[f\left(\tau_{\Delta} g\right)+\left(\tau_{\Delta} f\right) g\right]=\left(\tau_{\Delta} f\right)\left(\tau_{\Delta} g\right)-f g \text { a.e. on } \Delta \text {. }
$$

See [5, Chapter V] and [6, Chapter IV]. We first prove our main result in a special case.

Lemma. Let $v$ be a nonnegative function in $L^{1}(\Delta) \cap L^{\infty}(\Delta)$. Then the following statements are equivalent:

(i) for any $f \in L^{2}(\Delta) \cap L^{\infty}(\Delta)$,

$$
\int_{\Delta}\left|\left(\tau_{\Delta} f\right)(t)\right|^{2} v(t) d t \leq \int_{\Delta}|f(t)|^{2} v(t) d t
$$

(ii) $-\tau \Delta^{\nu} \in U(\Delta)$;

(iii) $v \in V(\Delta)$.

Proof. By (9), (i) is equivalent to having $-\int_{\Delta}\left(\tau_{\Delta}\left[f\left(\tau_{\Delta} f\right)^{*}+\left(\tau_{\Delta} f\right) f^{*}\right]\right) v d t$ $\geq 0$ for all $f \in L^{2}(\Delta) \cap L^{\infty}(\Delta)$, and by (8) this holds if and only if 


$$
\int_{\Delta}\left[f\left(\tau_{\Delta} f\right)^{*}+\left(\tau_{\Delta} f\right) f^{*}\right]\left(\tau_{\Delta} v\right) d t \geq 0
$$

for all $f \in L^{2}(\Delta) \cap L^{\infty}(\Delta)$. By [3, Theorem 8] this last condition is equivalent to (ii).

Assume that (ii) holds, so there exists $w$ in $R$ such that $-\left(\tau_{\Delta} v\right)(x)=$ $w(x+i 0)$ a.e. on $\Delta$. Then

$$
p(z)=\frac{1}{\pi} \int_{\Delta} \frac{v(t)}{t-z} d t+w(z), \quad z \in \Pi_{+},
$$

defines a function $p$ in $R$ such that

$$
p(x+i 0)=\left(\tau_{\Delta} v\right)(x)+i v(x)+w(x+i 0)=i v(x) \text { a.e. on } \Delta,
$$

and therefore (iii) holds.

Assume that (iii) holds, so there exists $p$ in $R$ such that $i v(x)=$ $p(x+i 0)$ a.e. on $\Delta$. Set

$$
w(z)=p(z)-\frac{1}{\pi} \int_{\Delta} \frac{v(t)}{t-z} d t, \quad z \in \cdot \Pi_{+} .
$$

Since $v$ is essentially bounded on $\Delta, \operatorname{Im} w(z)$ is bounded below in $\Pi_{+}$and hence $\exp (i w(z))$ is in $H^{\infty}$. But

$$
\operatorname{Im} w(x+i 0)=\chi_{\Delta c}(x) \operatorname{Im} p(x+i 0) \geq 0
$$

a.e. on $(-\infty, \infty)$, so the boundary function of $\exp (i w(z))$ is bounded by 1 a.e. on $(-\infty, \infty)$. Therefore $\exp (i w(z))$ is bounded by 1 in $\Pi_{+}$and $w \in R$. By construction

$$
w(x+i 0)=p(x+i 0)-\left(\tau_{\Delta} v\right)(x)-i \chi_{\Delta}(x) v(x)=-\left(\tau_{\Delta} v\right)(x) \text { a.e. on } \Delta,
$$

so (ii) holds and the proof is complete.

Theorem 4. A nonnegative measurable function $v$ on $\Delta$ satisfies

$$
\int_{\Delta}\left|\left(\tau_{\Delta} f\right)(t)\right|^{2} v(t) d t \leq \int_{\Delta}|f(t)|^{2} v(t) d t
$$

for all $f$ in $L^{2}(\Delta)$ such that $v f \in L^{2}(\Delta)$ if and only if $v \in V(\Delta)$.

Proof. Let $v$ be a nonnegative measurable function on $\Delta$, and let $\Delta_{1} \subseteq$ $\Delta_{2} \subseteq \cdots$ be Borel subsets of $\Delta$ such that $\bigcup_{1}^{\infty} \Delta_{n}=\Delta$ and the restriction $v_{n}$ of $v$ to $\Delta_{n}$ is in $L^{1}\left(\Delta_{n}\right) \cap L^{\infty}\left(\Delta_{n}\right)$.

If $v \in V(\Delta)$, then $v_{n} \in V\left(\Lambda_{n}\right)$ for every $n=1,2, \ldots$. By the Lemma,

$$
\int_{\Delta_{n}}\left|\left(\tau_{\Delta_{n}} f\right)(t)\right|^{2} v_{n}(t) d t \leq \int_{\Delta_{n}}|f(t)|^{2} v_{n}(t) d t
$$

for all $f \in L^{2}\left(\Delta_{n}\right) \cap L^{\infty}\left(\Delta_{n}\right)$. A routine approximation argument shows that (11) holds for all $f \in L^{2}(\Delta)$ such that $v f \in L^{2}(\Lambda)$.

Conversely suppose (11) holds for all $f \in L^{2}(\Delta)$ such that $v f \in L^{2}(\Delta)$. 
Then (12) holds for all $f \in L^{2}\left(\Delta_{n}\right) \cap L^{\infty}\left(\Delta_{n}\right), n=1,2, \ldots$ By the Lemma $v_{n} \in V\left(\Delta_{n}\right)$ and so there exists $w_{n} \in R$ such that $i v_{n}(x)=w_{n}(x+i 0)$ a.e. on $\Delta_{n}$. Since functions in $R$ are determined by their boundary functions on sets of positive measure, $w_{1}(z)=w_{2}(z)=\cdots$ in $\Pi_{+}$, and denoting this function by $w(z)$ we obtain $i v(x)=w(x+i 0)$ a.e. on $\Delta$. Thus $v \in V(\Delta)$.

\section{REFERENCES}

1. N. Aronszajn and W. F. Donoghue, Jr., On exponential representations of analytic functions in the upper half-plane with positive imaginary part, J. Analyse Math. 5 (1956/57), 321-388; Supplement, ibid. 12 (1964), 113-127. MR 29 \#6025.

2. M. Rosenblum and J. Rovnyak, Two theorems on finite Hilbert transforms, J. Math. Anal. Appl. 48 (1974), 708-720.

3. - Restrictions of analytic functions. I, Proc. Amer. Math. Soc. 48 (1975), 113-119.

4. - Restrictions of analytic functions. II, Proc. Amer. Math. Soc. 51 (1975), 335-343.

5. E. C. Titchmarsh, Introduction to the theory of Fourier integrals, 2nd ed., Clarendon Press, Oxford, 1948.

6. F. G. Tricomi, Integral equations, Interscience, New York, 1957. MR 20 \#1177.

DEPARTMENT OF MATHEMATICS, UNIVERSITY OF VIRGINIA, CHARLOTTESVILLE, VIRGINIA 22903 